\title{
The heat shock response in biology and human disease: a meeting review
}

The heat shock response first attracted the attention of molecular biologists because it offered an attractive experimental system for studying a small set of coordinately controlled genes. Further study showed that the heat shock response was actually an important homeostatic mechanism, enabling the cell to survive a variety of environmental stresses. The fundamental nature of this mechanism is underscored by the fact that the heat shock response is found in all animals, plants, and bacteria. The heat shock response is essentially a single cell response and, even for multicellular organisms, can be studied with homogeneous populations of cultured cells, circumventing the complexities of interactions between different cell types.

The many simple experimental systems for studying heat shock are yielding rapid advances in our understanding of the biology of both heat-shocked and normal cells; however, they have left open important questions about the role of heat shock in the complex physiology of multicellular organisms. For example, most animals have several mechanisms for regulating body temperature, ranging from the induction of fever to behavioral modifications. To what extent do changes in body temperature produce physiological effects by inducing the heat shock response at the level of individual cells? Answers to this question, among others, will undoubtedly be important in our understanding of disease. The importance of heat shock has been recognized for some time in the field of cancer therapy where hyperthermia is one component of treatment in many cases. For cancer therapy, the question is how best to overcome the heat shock defense of the cancer cell. To answer these questions will require expertise from a number of fields, including molecular and cell biology, physiology, oncology, and radiation biology. A recent Banbury Workshop, (November 9-13, 1987) organized by William Welch (Cold Spring Harbor Laboratory) and Richard Morimoto (Northwestern University) brought together a group of such specialists for the first time to consider the heat shock response. Several of the major themes that emerged from the Workshop are discussed below.

\section{Heat shock genes belong to gene families that have important roles in nonstressed cells}

In eukaryotes, the proteins encoded by the major heat shock-induced genes can be divided into three families. Although the apparent molecular weight of the proteins varies slightly from organism to organism, and even between different members of a gene family in the same organism, the strong conservation of both the nucleic acid and the amino acid sequences of these proteins allows easy identification of the members of each family. It was decided at the Workshop that each family would be denoted by a round number approximating its typical molecular weight. Thus, the hsp90 family includes proteins with an apparent molecular weight of 90 $\mathrm{kD}$ in mammals and $83 \mathrm{kD}$ in Drosophila and bacteria. The hsp70 family members include the mammalian 72 and $73-\mathrm{kD}$ proteins, the Drosophila 70 - and $68-\mathrm{kD}$ proteins, and the $70-\mathrm{kD}$ dnaK protein of $E$. coli. The hsp20 family includes proteins that range from 20 to $30 \mathrm{kD}$ in animals. In plants, the members of this family tend to be smaller than $20 \mathrm{kD}$. The hsp20 family does not show the strong sequence conservation seen in other families, but the members do share the pattern of hydrophobic regions. No bacterial member of this family has been identified.

A new eukaryotic heat shock protein was reported at the Workshop. This is a $58-\mathrm{kD}$ (in Tetrahymena, Richard Hallberg, Iowa State University) or $56-\mathrm{kD}$ (in mammals, Welch/ protein related to the GroEL protein of $E$. coli. The eukaryotic protein is found in mitochondria but is coded by a nuclear gene. Like its bacterial relative, the $56 / 58-\mathrm{kD}$ protein is present in nonstressed cells but synthesis is enhanced in stressed cells. The $E$. coli GroEL protein is one of two products from an operon whose activity is essential for growth above $20^{\circ} \mathrm{C}$ and for proper packaging of lambda phage (Takashi Yura, Kyoto University; Costa Georgopoulos, Utah). The analogies suggest that the $56 / 58-\mathrm{kD}$ eukaryotic protein might be involved in assembly or maintenance of a structure in the mitochondria.

Members of the hsp90 family are relatively abundant in nonstressed cells and synthesis is increased by stress. An important function of this protein described at the Workshop (Etienne Baulieu, INSERM, France) is its binding to the steroid hormone receptor. This binding appears to cap the DNA-binding site of the receptor. When the hormone binds to the receptor, the $90-\mathrm{kD}$ protein is released, freeing the DNA-binding site of the receptor and allowing interaction with DNA.

Eukaryotic organisms typically have several genes encoding proteins related to hsp70; some of these genes are expressed constitutively, others are induced by heat shock or other agents. Members of the $70-\mathrm{kD}$ family have an affinity for nucleotides and for fatty acids. In yeast a subset of the genes in the $70-\mathrm{kD}$ family have been shown to be necessary for translocation of several secreted and mitochondrial proteins; another member of the gene family encodes a mitochondrial protein (Elizabeth Craig, University of Wisconsin). Taken together 
with earlier evidence that the $70-\mathrm{kD}$ family in mammals includes the clathrin uncoating ATPase and the immunoglobulin heavy-chain binding protein, these results suggest that members of the $70-\mathrm{kD}$ family may play roles in several cellular compartments, acting by ATPdependent conformation changes or protein-protein interactions. A similar activity is proposed for the E. coli hsp70, which is called the DnaK protein. One role for this protein is facilitation of lambda phage replication. The DnaK protein acts by displacing the $\mathrm{P}$ protein so that the replication complex can proceed (Georgopoulos).

There are fewer hints as to possible functions for the hsp20 gene families. These proteins are notable in that they are found in 10-20S structures in the soluble fraction of the cell at control temperatures but move into perinuclear aggregates upon heat shock. This aggregation behavior is seen in tomatoes (Lutz, Nover, Halle, DDR), in Drosophila and mammals (Patrick Arrigo, Cold Spring Harbor Laboratory), and in chickens (Milton Schlesinger, Washington University).

\section{Cells are primed for rapid initiation of heat shock gene transcription}

The early studies on polytene chromosomes detected heat shock puffs within 5 min of shift to the high temperature. That evidence of rapid induction is consistent with the hypothesis that the response is a means of coping with sudden environmental change. A physical basis for this rapid transcriptional change can be seen in the recently isolated heat shock transcription factor (HSF). The Drosophila HSF is a $110-\mathrm{kD}$ protein that exists in an inactive state in control cells. Conversion of the HSF to an active form can be detected within $30 \mathrm{sec}$ after temperature increase. The activated form binds to the regulatory DNA sequence (HSE) of the heat shock promoter and induces transcription. Although the mechanism of activation of the HSF is not yet understood, it appears not to require protein synthesis; multiple rounds of activation and inactivation are obtained when cells are alternately heat-shocked and allowed to recover in the presence of cycloheximide (Carl Wu, National Institutes of Health). The yeast HSF is a $150-\mathrm{kD}$ protein that is bound to the HSE in both control and heatshocked cells. The yeast HSF undergoes multiple phosphorylations in response to stress. It is proposed that these phosphorylations are the means by which this factor is activated (Peter Sorger, University of Cambridge). In view of the very strong conservation of the HSE sequence, the differences between the yeast and Drosophila HSF are puzzling. John Lis (Cornell University) reported that, in nonstressed cells, the Drosophila hsp70 and hsp26 genes have a transcriptionally engaged polymerase apparently stalled within the first $30 \mathrm{nu}-$ cleotides. This too might be a means for speeding the induction of transcription. The human hsp70 gene is a member of several regulatory pathways since it can be induced independently of heat shock by agents such as serum starvation, passage through the $G_{1} / S$ boundary, and heavy metals. The 170 nucleotides $5^{\prime}$ to the tran- scription start contain separable regulatory sequences for each of the inducers (Morimoto).

Prokaryotic cells also have a preexisting heat shock transcription factor, $\sigma^{32}$. This protein is made in control cells but turns over rapidly when cells are not stressed. Heat shock increases the amount of $\sigma^{32}$ by stabilizing its turnover and by increasing its translation (Carol Gross, University of Wisconsin; Frederick Neidhardt, University of Michigan). At very high temperatures, yet another $\sigma$-factor is induced which also acts in transcription of heat shock genes (Gross).

The isolation of HSF and $\sigma^{32}$ puts us one step closer to understanding how the environmental stress is translated into changes in cellular activity, but we still do not know how many steps remain to be detected. It has been proposed that the stress produces denatured or aberrant proteins which then induce the heat shock response. The La protease is induced by heat shock in $E$. coli (Alfred Goldberg, Harvard University). In eukaryotes ubiquitin synthesis increases during heat shock (Schlesinger; Alex Varshavsky, Massachusetts Institute of Technology). In some cases, heat shock induction has been shown to be associated with damaged proteins; however, there are also cases where heat shock is induced under conditions that should not be detrimental to proteins, as well as cases where protein damage does not induce heat shock. Thus, it seems that, although protein damage may sometimes trigger heat shock, it is not the only trigger for this response. A promising approach for identifying other elements of the trigger was reported by Jose Bonner (Indiana University). He has used deletions to screen a large part of the Drosophila genome for loci that are necessary for induction of heat shock. The three regions that have been detected may well contain genes for elements of the trigger; however, more work will be needed to determine whether this is so.

\section{Preinduction of the heat shock genes can afford thermoprotection of many different cellular processes}

The evolutionary conservation of the heat shock response is strong evidence that the response is beneficial to cells. The most obvious advantage conferred by heat shock is thermotolerance. Induction of a low level of heat shock synthesis allows the cell or the organism to withstand later temperatures that would otherwise produce severe damage or death. This induced thermotolerance is transient and is lost over a period of hours or days. The decay of the thermotolerant state depends on the time and temperature of its development and on other conditions. For example, experimental depletion of polyamines leads to more rapid loss of thermotolerance, although it does not affect the induction of the tolerance (Eugene Gerner, University of Arizona).

There is general agreement that thermotolerance depends on preinduction of heat shock, but it is not clear which elements of the response are most important. Nor is it clear what the physical basis of the thermotolerance is. Many cellular activities and structures are changed by the heat stress; in thermoprotected cells all of these 
remain normal at temperatures several degrees above the temperatures that cause alteration in unprotected cells. Stress-induced changes for which thermoprotection has been demonstrated include: blockage of RNA splicing (Susan Lindquist, University of Chicago), loss of translational activity, collapse of the intermediate filaments, the movement of hsp70 into and out of the nucleus, the change in nucleolar morphology (Welch), heat radiosensitization (heat-induced increase in killing by ionizing radiation), and loss of DNA polymerase alpha and beta activities (William Dewey, University of California, San Francisco). None of these specific thermoprotections has been identified as primary to any of the others. At this point it almost appears that thermoprotection is acting by setting some cellular thermostat a few degrees higher than normal. One component of thermotolerance may involve the synthesis of new ribosomes. Cells in which the recovery of new ribosomal RNA synthesis was delayed by inhibitors did not display thermotolerant recovery (John Subjeck, Roswell Park Memorial Institute).

Understanding thermotolerance has important medical implications. The combination of heat and radiation is more effective than radiation alone in the treatment of tumors. The effectiveness of the heat treatment is diminished when thermotolerance is induced either by previous heat treatment or by certain drugs. Transient thermotolerance is distinguished from long term thermoresistance. Cell lines selected for thermoresistance retain the capacity to develop thermotolerance /George Hahn, Stanford University). An accurate measure of the thermotolerance of the targeted cells would be useful in determining treatment schedules and chemotherapy.

Heat shock can be induced in almost every cell type; the notable exceptions are spermatocytes and the first few cells in an embryo (Debra Wolgemuth, Columbia University). However, there appear to be cell-type-specific differences in the sensitivity of heat shock induction of the response. Some regions of the mammalian brain show high levels of hsp70 in control animals. A temperature increase of $2^{\circ} \mathrm{C}$ (Ian Brown, University of Toronto) or transient ischemia (Thaddeus Nowak, National Institutes of Health) caused strong induction of hsp synthesis in a set of cell types that had only partial overlap with those showing constitutive synthesis. It is interesting that the CA1 neurons, which die after brief ischemia, showed low levels of hsp70, while the CA3 neurons, which are spared, had much higher levels. Rats treated with a thermotolerance-inducing regime at $41^{\circ} \mathrm{C}$ showed decreased killing of retinal photoreceptors by light. Again, the protection correlated with the presence of hsp70 (Michael Tytell, Bowman Gray Medical School, Wake Forest University).

Severe heat shocks can induce a number of developmental defects. The type of defect induced depends on the point in its development when the organism received the stress. In both mammals (Larry Hightower, University of Connecticut) and Drosophila (Nancy Petersen, University of Wyoming), induced thermotolerance can prevent these defects. The conditions required for developmental thermotolerance are the same as those for other forms of thermotolerance, suggesting similar molecular mechanisms.

\section{Heat shock proteins may play unexpected roles in the immune response}

A recent and unexpected finding is that many antibodies and T-lymphocyte responses induced by mycobacterial infection are recognizing epitopes on heat shock proteins of the pathogen. Similar results have been seen in a wide variety of bacterial and parasitic infections. It was suggested that the immunogenicity of the heat shock proteins might be due both to a strong induction of heat shock in the pathogen when it invades the host and to the conserved structures of the heat shock proteins. Because of this conservation, the immune system of the host has a good probability of a previous encounter with epitopes similar enough to prime a strong response. The conserved nature of the heat shock proteins also increases the probability of autoimmune responses which add to the pathology of the disease (Douglas Young, Hammersmith Hospital, London).

This Banbury Workshop has demonstrated the increasing number of links between the study of heat shock and other fields of biology. Given the fundamental nature of this cellular response, we can expect more links in the future.

\section{Mary Lou Pardue}

Department of Biology

Massachusetts Institute of Technology

Cambridge, Massachusetts 02139 USA 


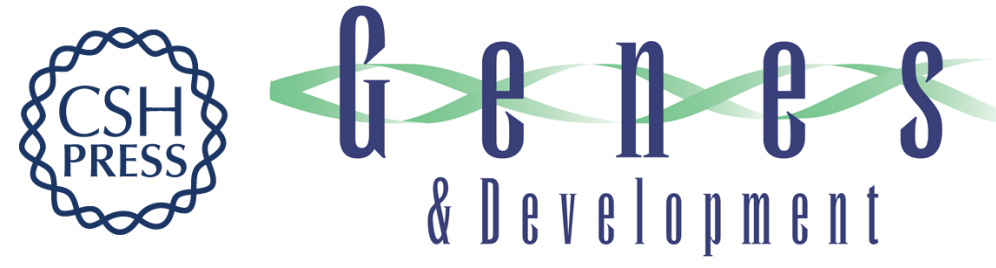

\section{The heat shock response in biology and human disease: a meeting review}

Mary Lou Pardue

Genes Dev. 1988, 2:

Access the most recent version at doi:10.1101/gad.2.7.783

\section{License}

Email Alerting Receive free email alerts when new articles cite this article - sign up in the box at the top Service right corner of the article or click here.

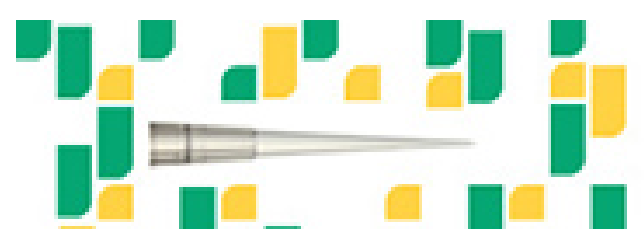

Focused on your science.

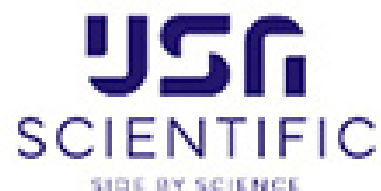

